\title{
E-cadherin mRNA expression analysis in evaluating the natural history of urothelial bladder cell carcinoma: Results from a long-term follow-up study
}

\author{
TOMMASO CAI ${ }^{1}$, MAURO PIAZZINI $^{2}$, GABRIELLA NESI $^{3}$, ILARIA TADDEI $^{2}$, IACOPO SARDI $^{4}$, \\ BEATRICE DETTI $^{5}$, NICOLA MONDAINI ${ }^{1}$, MAURIZIO DAL CANTO ${ }^{1}$ and RICCARDO BARTOLETTI ${ }^{1}$ \\ ${ }^{1}$ Department of Urology, ${ }^{2}$ Medical Genetics Unit, Department of Clinical Physiopathology, Departments of ${ }^{3}$ Pathology \\ and Oncology, ${ }^{4}$ Pediatrics (Onco-Hematology Unit), ${ }^{5}$ Radiation Oncology, University of Florence, Florence, Italy
}

Received July 26, 2006; Accepted October 6, 2006

\begin{abstract}
Many studies have indicated that the E-cadherin (E-CAD) expression loss is associated with the loss of cellular differentiation and increased cellular invasiveness and can be correlated with poor prognosis in urothelial carcinoma (UC) of the urinary bladder. The aim of this study was to define the role of E-CAD mRNA expression on recurrence, progression and survival in UC of the urinary bladder over a long follow-up period. From 30 patients with bladder UC, enrolled in our previous study, 27 were selected for this study. All patients were re-analyzed in terms of clinical and tumor characteristics, tumor pathological analysis and tumor E-CAD mRNA expression. The data were correlated to 12-year follow-up results. Significant correlations between stage $(\mathrm{p}=0.002)$, grade $(\mathrm{p}=0.008)$ and E-CAD mRNA expression were reported. E-CAD did not show any correlation in predicting recurrence or progression in bladder UC. The survival analysis demonstrated a significant relationship ( $\mathrm{p}=0.019)$ between patients with expressed E-CAD mRNA levels and cancer-specific survival. Multivariate analysis demonstrated that expression of E-CAD mRNA levels is an independent prognostic factor in terms of cancerspecific survival in UC of the urinary bladder $(\mathrm{p}=0.002)$. Our study is the first to demonstrate that mRNA extraction and Northen blot analysis is to be considered a reliable method to evaluate E-CAD mRNA levels for predicting survival rate in patients affected by urothelial bladder cancers. We stress that a long follow-up period is needed to evaluate the role of molecular factors in predicting prognosis in patients affected by bladder UC.
\end{abstract}

Correspondence to: Dr Tommaso Cai, Department of Urology, University of Florence, Via dell'Antella 58, I-50011 Bagno a Ripoli, Florence, Italy

E-mail: ktommy@libero.it

Key words: E-cadherin, urothelial cell carcinoma, bladder cancer, Northern blot analysis, mRNA, recurrence, progression

\section{Introduction}

Cadherins are transmembrane proteins (1) associated with cytoplasmic molecules, which using a $\mathrm{Ca}^{++}$dependent mechanism, are involved in cell-to-cell adhesion and cell-to-stroma interaction (2). The cadherin family includes N-, P- and E-cadherin (E-CAD) but only E-CAD is expressed in all epithelial tissues (1). E-CAD, a 120-kD glycoprotein located on chromosome 16 (3), is associated with intracellular proteins called catenins that mediate between extra-cellular signals and cytoskeleton microfilaments (4). The functions of E-CAD are related to tumor suppression and are generally down-regulated in epithelial tumor development and diffusion (5). Many authors have reported that loss of E-CAD expression is associated with loss of cellular differentiation and increased cellular invasiveness (6). Moreover, E-CAD expression loss is related to high grade and advanced stage in many neoplasms, such as breast $(7)$ and colorectal $(8,9)$ cancers and urologic tumors $(4,10)$. Recent studies have demonstrated that loss of normal E-CAD expression also correlates with poor prognosis in urothelial carcinoma (UC) of the upper urinary tract (11) and urinary bladder (5). By using immunohistochemical analysis, Byrne et al showed in 77 patients undergoing radical cystectomy for invasive bladder cancer that loss of E-CAD expression is associated with an increased risk of disease progression (12). However, several authors have suggested that E-CAD is not an independent prognostic factor in UC of the urinary bladder (13). Tsukino et al recently demonstrated a significant correlation between E-CAD polymorphism and the occurrence of urothelial cancer, although they did not show any relationship with disease progression (1). The absence of correlation between loss of normal E-CAD expression and prognosis in UC may be due to a short follow-up period often too limited for the prediction of patient outcome or else to a lack of statistical power in the various analyses. In UC patients, it is thought appropriate to carry out a long-term follow-up, so as to better determine the prognostic factors of recurrence, progression and diseasespecific mortality $(14,15)$. The alteration site of E-CAD function has not been sufficiently investigated or identified to date and we have no information regarding type and site of E-CAD function loss. The E-CAD complex can be regulated 
at multiple levels through its different elements (e.g., mutations in cadherin or catenin genes, mRNA stability, phosphorylation of $\beta$-catenin, intra- or extracellular association with other proteins) (3). In a previous study, we analyzed the role of E-CAD gene alterations and E-CAD mRNA levels in 30 bladder carcinoma patients, and suggested that the E-CAD gene may be involved in bladder carcinogenesis and clinical progression (16). This study, however, did not provide any relationship between E-CAD mRNA expression and prognosis in UC patients, possibly owing to a follow-up period which was too short. The aim of the current study is to evaluate the prognostic role of E-CAD mRNA expression in patients with urothelial bladder carcinoma treated with surgery and adjuvant chemotherapy over a long follow-up period.

\section{Patients and methods}

Study design. To evaluate the prognostic role of E-CAD mRNA expression in UC of the urinary bladder, we re-analyzed all patients enrolled in our previous study (16), taking into account patient and tumor clinical characteristics, tumor pathologic parameters and E-CAD mRNA expression. Data collected for each case were checked before being linked to vital status information at 12-year follow-up.

Inclusion and exclusion criteria. Only those patients with complete clinicopathologic and molecular data and only those who had shown urothelial bladder cell carcinomas on pathological analysis were enrolled in the present study. Cases with no tissue blocks available for further histopathologic analysis were excluded from the study.

Study population. Of the 30 patients enrolled in the previous study (16), 27 were finally selected for this study, 24 (88.8\%) were male and $3(11.2 \%)$ female (median age 68.7 years). Three cases of squamous cell carcinoma of the urinary bladder were excluded from the study, in agreement with inclusion criteria described above. Sixteen $(59.25 \%)$ had recurrent tumors, while $11(40.75 \%)$ were newly diagnosed with bladder carcinoma. Among recurrent cancers, no patient reported disease progression. Thirteen patients had a single lesion, 6 had 2 lesions, 6 had 4 lesions and 2 had 5 lesions. Mean tumor diameter was $2.2 \pm 0.7 \mathrm{~cm}$ (range $1-6 \mathrm{~cm}$ ). Transurethral resection of bladder tumor (TURBT) was performed in 14 patients and radical cystectomy (RC) with urinary diversion in 13. Patients who had undergone RC had initially been treated with TURBT that had shown $\geq \mathrm{pT} 2$ bladder cancer. Six out of 14 patients with superficial UC were classified as low-risk, 5 as intermediate risk and 3 as high-risk, according to the European Association of Urology (EAU) Working Group on Oncological Urology classification (17). Among those patients undergoing TURBT, 7 received adjuvant treatment for 6 weeks with weekly Bacillus Calmette-Guérin (BCG) instillations at an average interval of 21 days after the operation. Of the patients undergoing $\mathrm{RC}$, only 3 received adjuvant systemic chemotherapy and 2 systemic chemotherapy with the addition of local radiotherapy for palliative pain treatment.

Histopathology. All available hematoxylin and eosin stained slides of primary tumors, as well as their recurrences, were re- evaluated by one dedicated pathologist without knowledge of the clinical status. Specimens were pathologically staged according to the International Union Against Cancer (UICC) 2002 issue of the Tumor, Nodes, Metastasis System (18). Tumor grade was assessed following the 1973 World Health Organization (WHO) classification (19).

Molecular analysis. The laboratory methods used are detailed in our previous report (16). Briefly, several tumor tissue samples were taken from each patient. Some were sent for pathologic examination and some were immediately frozen, using liquid nitrogen with a sterile technique for molecular analysis. Blood samples were taken before surgery in order to obtain normal DNA content from lymphocyte blood cells. In each case, samples were also taken from macroscopically normal-appearing mucosa at a distance from the tumor(s) during TURBT or RC in order to create a tissue control group. These samples were bisected and used for both pathological assessment (to exclude the presence of neoplastic cells) and molecular evaluation.

Blot analysis. All tissue samples were subjected to total-mRNA extraction and Northern blot analysis, marked with specific probes for E-CAD and evaluated by gel-electrophoresis on polyacrylamide and autoradiographic printing, as we have previously described $(16,20)$.

RNA extraction and Northern blotting. Total-RNA was extracted by the guanidinium isothiocyanate method (21). Samples of $20 \mu \mathrm{g}$ were separated by electrophoresis on $1 \%$ agarose gel containing 2.2 $\mathrm{M}$ formaldehyde and transferred to a nylon membrane (Hybond-N, Amersham). After $2 \mathrm{~h}$ at $80^{\circ} \mathrm{C}$, the resulting filters were prehybridized for $1-2 \mathrm{~h}$ at $42^{\circ} \mathrm{C}$ in a hybridization buffer containing $50 \%$ deionized formamide, $5 X$ Denhardt's solution, 1X SSPE and $100 \mu \mathrm{g} / \mathrm{ml}$ denatured salmon sperm DNA for $18-24 \mathrm{~h}$ at $42^{\circ} \mathrm{C}$ and washed according to the manufacturer's instructions. We used PCR product fragments as E-CAD cDNA probes obtained by amplification of sequences from NIH-GenBank using the following primers: E-CAD FW, 5'-CCTTCACAGCAGAACTAACA-3'; E-CAD RV, 5'-CGCTTTCAGATTTTCATCAA-3'; and those purified by QIAquick Gel Extraction kit (Qiagen, Valencia, USA). Each probe was labeled with $\left[\alpha-{ }^{32} \mathrm{P}\right]-\mathrm{dCTP}$, using random priming reaction (Boehringer Mannheim, Germany). We used the glyceraldehyde-3-phosphate dehydrogenase (GAPDH) probe obtained by PCR using the following primers: GAPDH FW, 5'-CCATGGAGAAGGCTGGGG-3'; GAPDH RV, 5'-CAAA GTTGTCATGGATGACC-3' as an internal control in order to adjust for differences in the amount of RNA loaded in each lane. Filters were exposed for autoradiography to Kodak Xar-5 film (Eastman Kodak, Rochester, NY) for 1-6 days at $-80^{\circ} \mathrm{C}$ (22).

Test reading and interpretation. The expression level of both mRNAs was quantified by densitometry of autoradiograms using the GS-670 Imaging Densitometer (Bio-Rad, CA, USA). According to the recommendations of Pertschuk et al, we considered the densitometric percentage of the autoradiographic signal (23). In order to exclude possible densitometric signal errors due to background and/or other aspecific 


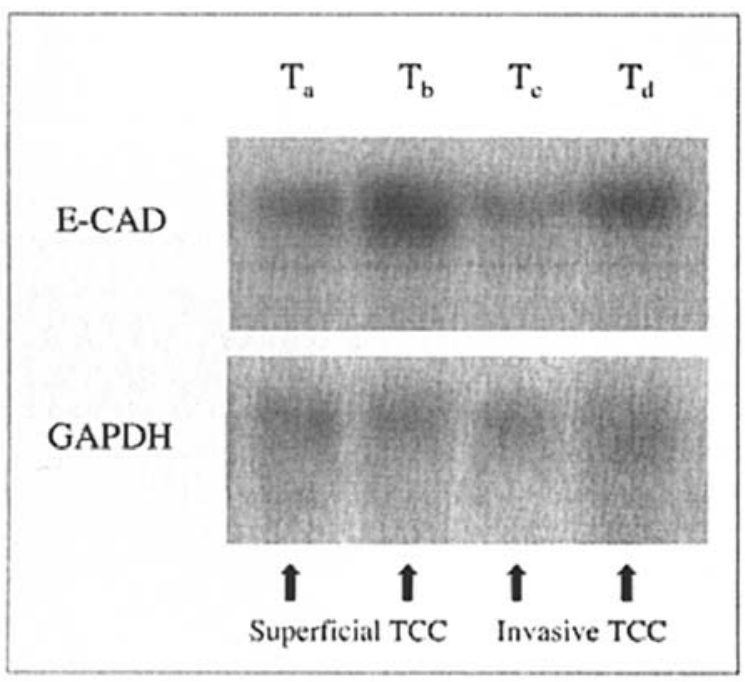

Figure 1. Northern blot analysis. Expression of E-cadherin mRNA in tissue from superficial and invasive bladder patients. The GAPDH mRNA band is shown as a control for equal RNA loading. The autoradiographs are representative of 4 tumors, 2 superficial $\left(\mathrm{T}_{\mathrm{a}}, \mathrm{T}_{\mathrm{b}}\right)$ and 2 invasive urothelial bladder cell carcinomas $\left(\mathrm{T}_{\mathrm{c}}, \mathrm{T}_{\mathrm{d}}\right)$.

phenomena, the intensity of mRNA expression was compared to that of a control gene (GAPDH) (Fig. 1).

Patient follow-up. All patients underwent a specific schedule follow-up on the basis of clinical, instrumental and pathologic characteristics of the bladder tumor. Follow-up was performed at a mean period of 12 years after surgical treatment. All patients were contacted by telephone by a urologist and information concerning status at follow-up, adjuvant therapy, recurrence and progression was collected. Recurrence was determined by papillary formations protruding into the bladder lumen and detected on cystoscopy, or by the presence of neoplastic tissue diagnosed after vesical biopsy. Progression was defined as the increase in tumor stage or grade.

Statistical analysis. Pearson's coefficient was adopted to evaluate the correlation between the different parameters in both superficial and invasive bladder cancer patients, and Fisher's exact test was used to assess statistical significance with $\mathrm{p}<0.05$ accepted as significant. Two-sided tests of significance were performed for all analyses. Kaplan-Meier survival curves were also used to estimate survival, and survival probability distributions were compared with the log-rank test (Mantel-Cox). Univariate and multivariate analyses were also used to identify the role of each factor in terms of prognostic evaluation. All statistical analyses were performed using SPSS 11.0 for Apple Macintosh (SPSS, Inc., Chicago, IL).

\section{Results}

Histopathological evaluation. Pathologic examination showed that stratification according to stage and grade was as follows: 11 pTa (7 G1, 1 G2, 3 G3), 3 pT1 G3, 1 pT2 G3, 9 pT3 G3, 3 pT4 G3. Lymph node invasion was found in 3 pT3 G3 and in 2 pT4 G3. No carcinoma in situ (CIS) associated with superficial or invasive bladder cancer was
Table I. Follow-up results in 27 bladder UC patients according to stage and grade.

\begin{tabular}{cccccc}
\hline & $\begin{array}{c}\text { ANED } \\
\text { no. }(\%)\end{array}$ & $\begin{array}{c}\text { AREC } \\
\text { no. }(\%)\end{array}$ & $\begin{array}{c}\text { DUC } \\
\text { no. }(\%)\end{array}$ & $\begin{array}{c}\text { DUN } \\
\text { no. }(\%)\end{array}$ & Total \\
\hline Stage & & & & & \\
pTa & $6(54.5)$ & $1 \quad(9.1)$ & $2(18.2)$ & $2(18.2)$ & 11 \\
pT1 & & & $2(66.6)$ & $1(33.4)$ & 3 \\
pT2 & & & $1(100)$ & & 1 \\
pT3 & & & $9(100)$ & & 9 \\
pT4 & & & $3(100)$ & & 3 \\
Total & 6 & 1 & 17 & 3 & 27 \\
Grade & & & & & \\
G1 & $6(85.7)$ & $1(14.3)$ & & & 7 \\
G2 & & & $16(84.2)$ & $3(15.8)$ & 19 \\
G3 & & & 11 & 3 & 27 \\
Total & 6 & 1 & & & \\
\hline
\end{tabular}

Follow-up results in 27 urothelial bladder cell carcinoma patients according to stage and grade. UC, urothelial carcinoma; ANED, alive with no evidence of disease; AREC, alive with previous single or multiple recurrences; DUC, died of urothelial bladder cell carcinoma progression; DUN, died for unrelated reasons. Data in parentheses are percentages.

found. At the pathologic review, 3 pTa G2 were re-classified as pTa G3.

Molecular findings. Among all patients, 10 (37\%) demonstrated E-CAD mRNA expression, while 17 (63\%) were negative at this analysis. Regarding stage and grade, 9 of the E-CAD mRNA positive patients were pTa ( 7 G1, 1 G2, 1 G3) and 1 pT4 G3. Among E-CAD mRNA negative patients, 2 were pTa (2 G3), 3 pT1 G3, 1 pT2 G3, 9 pT3 G3 and 2 pT4 G3. These findings are in line with data from the literature $(12,13,24)$. All samples of normal-appearing mucosa distant from superficial bladder tumors were positive on E-CAD analysis, while among the 14 invasive tumors, 11 were negative and 3 were positive. By comparing molecular analysis results with pathologic stage and grade, we found a significant correlation (stage, $\mathrm{p}=0.002$; grade, $\mathrm{p}=0.008$ ) demonstrating that superficial bladder tumors are more likely to be E-CAD positive than invasive ones.

Clinical and instrumental follow-up. The information on vital status at 12 years was available for all patients (range, 11-13 years). Six $(22.2 \%)$ patients were alive with no evidence of disease (ANED), while $17(62.9 \%)$ died of disease progression, $1(3.7 \%)$ was alive with previous multiple recurrences invariably treated with transurethral resection (equal or lower stage and grade) and $3(11.2 \%)$ died of unrelated causes. The follow-up results at 12 years, according to stage and grade, are shown in Table I. Among the 6 ANED patients, 4 (66.6\%) showed E-CAD mRNA expression and among the 14 who 


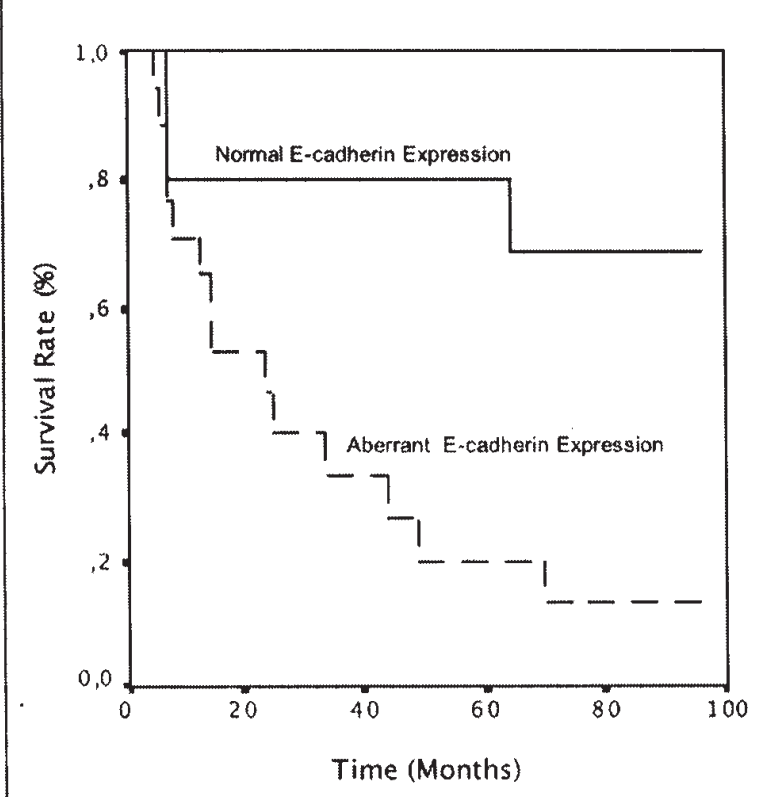

Figure 2. Kaplan-Meier survival curve in all enrolled patients (— E-CAD mRNA expressed; ------, E-CAD mRNA not expressed). Kaplan-Meier estimates of the probability of bladder cancer-related survival according to E-cadherin expression $(\mathrm{p}=0.019)$. died of disease progression, 11 (78.5) did not express E-CAD mRNA.

Recurrence and progression. No correlation was found between number $(\mathrm{p}=0.643)$, time relapse $(\mathrm{p}=0.097)$ or other parameters of recurrence or progression $(p=0.078)$ and E-CAD mRNA expression. E-CAD was not associated with recurrence or progression in UC of the urinary bladder.

Survival analysis. Survival analysis, performed by using Kaplan-Meier curves on all enrolled UC patients, showed a significant relationship $(\mathrm{p}=0.019)$ between patients with expressed E-CAD mRNA and cancer-specific survival (Fig. 2). The outcome analysis, carried out by dividing all patients by superficial or invasive UC features, did not demonstrate any significant correlation with cancer-specific survival ( $\mathrm{p}=0.219$ and 0.415 , respectively). However, on multivariate analysis, in addition to stage $(\mathrm{p}<0.001)$ and grade $(\mathrm{p}=0.003)$, E-CAD mRNA expression proved to be independent prognostic factors in terms of cancer-specific survival in UC of the urinary bladder $(\mathrm{p}=0.002)$ (Table II).

\section{Discussion}

The role of cell-to-cell adhesion and cell-to-stroma interaction and their complex regulation mechanisms have been

Table II. Multivariate analysis of factors affecting survival in 27 UC patients.

\begin{tabular}{|c|c|c|c|c|}
\hline $\begin{array}{l}\text { Categories } \\
\text { (variables) }\end{array}$ & $\begin{array}{c}\text { No. of patients } \\
(\%)\end{array}$ & $\begin{array}{l}\text { No. of survival } \\
\text { patients }(\%)\end{array}$ & $\begin{array}{c}\text { Survival time at } 12 \text { years } \\
\text { follow-up (mean) - (95\% C.I.) }\end{array}$ & $\begin{array}{c}\text { Multivariate analysis } \\
\text { p-value }\end{array}$ \\
\hline \multicolumn{5}{|l|}{ Presentation } \\
\hline First occurrence & $11(40.8)$ & $3(27.2)$ & $11.8(10.9-12.4)$ & 0.561 \\
\hline Recurrence & $16(59.2)$ & $3(18.7)$ & $11.3(10.2-12.1)$ & \\
\hline \multicolumn{5}{|l|}{ No. of lesions } \\
\hline Single & $13(48.1)$ & $4(30.7)$ & $11.6(10.3-12.2)$ & 0.751 \\
\hline Multiply & $14(51.9)$ & $2(14.2)$ & $11.9(11.0-12.6)$ & \\
\hline \multicolumn{5}{|l|}{ Diameter of lesions } \\
\hline$<3.0 \mathrm{~cm}$ & $18(66.6)$ & $3(16.6)$ & $11.8(10.9-12.4)$ & 0.612 \\
\hline$\geq 3.0 \mathrm{~cm}$ & $9 \quad(33.4)$ & $3(33.3)$ & $11.3(10.2-12.1)$ & \\
\hline \multicolumn{5}{|l|}{ Stage } \\
\hline $\mathrm{pTa}$ & 11 (40.7) & $6(54.5)$ & $11.7(10.4-12.6)$ & \\
\hline pT1 & $3(11.1)$ & - & & \\
\hline pT2 & $1 \quad(3.7)$ & - & & $<0.001$ \\
\hline pT3 & $9 \quad(33.4)$ & - & & \\
\hline pT4 & $3(11.1)$ & - & & \\
\hline \multicolumn{5}{|l|}{ Grade } \\
\hline G1 & $7 \quad(25.9)$ & $6(85.7)$ & $11.7(10.4-12.6)$ & \\
\hline G2 & $1 \quad(3.7)$ & - & & 0.003 \\
\hline G3 & $19(70.4)$ & - & & \\
\hline \multicolumn{5}{|l|}{ E-CAD mRNA } \\
\hline Expressed & $10(37.0)$ & $4(66.6)$ & $11.4(10.3-12.1)$ & 0.002 \\
\hline Non-expressed & $17(63.0)$ & $2(11.7)$ & $11.9(11.1-12.6)$ & \\
\hline
\end{tabular}

Multivariate analysis of factors affecting survival in 27 urothelial bladder cell carcinoma patients. UC, urothelial carcinoma. 
thoroughly analyzed to explain normal epithelial tissue structure control (25). E-CAD is a protein involved in this control mechanism and its altered expression in bladder tumors is associated with the lack of epithelial structure and the degree of invasiveness and diffusion (26). Published data on UC show that E-CAD expression loss correlates with advanced stage, high grade (27), increased recurrence rate (28), high progression probability and poor prognosis (29), although not all authors have found E-CAD expression significantly correlating with disease progression and poor prognosis (30). In 69 pT1 urothelial cancers, Mahnken et al reported that E-CAD immunoreactivity displays an important prognostic role in predicting recurrence-free survival ( $\mathrm{p}=0.016)$ but not progression-free survival ( $\mathrm{p}=0.071$ ) (31). The role of E-CAD function loss with regard to disease recurrence or progression is debatable. The complex E-CAD/catenin regulator mechanism can be altered at multiple levels: DNA expression, DNA transcription, mRNA expression and protein synthesis. In the present study, we investigated the role of E-CAD mRNA expression by using mRNA extraction and Northern blot analysis to predict recurrence rate, disease progression and cancer-specific survival in UC patients. This is the first study to explore loss of E-CAD function due to the lack of mRNA expression and to correlate these findings with data from a long follow-up period. We evaluated 27 patients with urothelial bladder carcinoma, comparing the E-CAD mRNA expression with disease recurrence or progression at a 12-year follow-up. A statistically significant inverse correlation was found between E-CAD mRNA expression and pathologic parameters (stage, $\mathrm{p}=0.002$ and grade, $\mathrm{p}=0.008$ ), confirming that superficial bladder tumors had higher frequency of E-CAD mRNA expression than invasive ones (16). These findings are in line with data from the literature highlighting the distinct association of decreased E-CAD expression with high tumor grade and advanced stage (27). No correlation between E-CAD mRNA expression and recurrence rate $(\mathrm{p}=0.643)$ or progression $(\mathrm{p}=0.078)$ was found. This may be due to the relatively small number of cases of superficial UC enrolled in the study, even though the followup period considered was long. The outcome analysis, performed by using Kaplan-Meier curves, demonstrated a significant relationship $(\mathrm{p}=0.019)$ between patients with $\mathrm{E}-\mathrm{CAD}$ mRNA expression and cancer-specific survival, suggesting that the expression of E-CAD mRNA is an important prognostic factor in urothelial bladder cancers. These results were validated on multivariate analysis identifying E-CAD mRNA as an independent prognostic factor along with stage and grade. This study highlights the important role of E-CAD mRNA expression in predicting the clinical behavior of bladder carcinomas and in planning a treatment tailored for each patient. Molecular analysis also demonstrated that the normal-appearing mucosa distant from superficial bladder tumors was E-CAD positive, while among invasive tumors 11 out of 14 were negative and 3 out of 14 were positive. These findings suggest that structural alterations or carcinogenetic processes may involve the whole bladder, implying that bladder cancer could be an 'organ disease', despite its solitary or episodic appearance, as we have previously hypothesized in other studies $(15,20)$.

In conclusion, we report the significant prognostic role of E-CAD mRNA expression in UC of the urinary bladder. This is the first study to demonstrate that mRNA extraction and Northern blot analysis are reliable methods for assessing ECAD mRNA expression in the prediction of survival in bladder cancer patients. The study also emphasizes the fact that a long follow-up period is needed to evaluate the prognostic significance of molecular factors in urothelial bladder cancer.

\section{Acknowledgements}

Special thanks to Professor John Denton for manuscript language revision.

\section{References}

1. Tsukino H, Kuroda Y, Nakao H, et al: E-cadherin gene polymorphism and risk of urothelial cancer. Cancer Lett 195: 53-58, 2003.

2. Garcea G, Neal CP, Pattenden CJ, Steward WP and Berry DP: Molecular prognostic marker in pancreatic cancer: a systematic review. Eur J Cancer 41: 2213-2236, 2005.

3. Mareel M, Boterberg T, Noe V, et al: E-cadherin/catenin/cytoskeleton complex: a regulator of cancer invasion. J Cell Physiol 173: 271-274, 1997

4. Okegawa T, Li Y, Pong RC and Hsieh JT: Cell adhesion proteins as tumor suppressors. J Urol 167: 1836-1843, 2002.

5. Bryan RT, Hussain SA, James ND, Jankowski JA and Wallace DMA: Molecular pathways in bladder cancer: Part 2. BJU Int 95: 491-496, 2005.

6. Frixen UH, Behrens J, Sachs M, Eberle G, et al: E-cadherinmediated cell-cell adhesion prevents invasiveness of human carcinoma cells. J Cell Biol 113: 173-185, 1991.

7. Oka H, Shiozaki H, Kobayashi K, et al: Expression of E-cadherin cell adhesion molecules in human breast cancer tissue and its relationship to metastasis. Cancer Res 53: 1696-1701, 1993.

8. Nigam AK, Savage FJ, Boulos PB, Stamp GWH, Liu D and Pignatelli M: Loss of cell-cell and cell-matrix adhesion molecules in colorectal cancer. Br J Cancer 68: 507-514, 1993.

9. Bendardaf R, Elzagheid A, Lamlum H, Ristamaki R, Collan Y and Pyrhonen S: E-cadherin, CD44s and CD44v6 correlate with tumour differentiation in colorectal cancer. Oncol Rep 13: 831-835, 2005.

10. Umbas R, Isaacs WB, Bringuier PP, et al: Decreased E-cadherin expression is associated with poor prognosis in patients with prostate cancer. Cancer Res 54: 3929-3933, 1994.

11. Fromont G, Rouprêt M, Amira N, et al: Tissue microarray analysis of the prognostic value of E-cadherin, Ki67, p53, p27, survivin and MSH2 expression in upper urinary tract transitional cell carcinoma. Eur Urol 48: 764-770, 2005.

12. Byrne RR, Shariat SF, Brown R, et al: E-cadherin immunostaining of bladder transitional cell carcinoma, carcinoma in situ and lymph node metastases with long-term follow-up. J Urol 165: 1473-1479, 2001.

13. Hong R, Pu Y, Hsieh T, Chu JS and Lee WJ: Expressions of E-cadherin and exon V6-containing isoforms of CD44 and their prognostic values in human transitional cell carcinoma. J Urol 153: 2025-2028, 1995.

14. Heney NM, Ahmed S, Flanagan MJ, et al: Superficial bladder cancer: progression and recurrence. J Urol 130: 1083-1086, 1983

15. Cai T, Margallo E, Nesi G, Giubilei G, Rizzo M and Bartoletti R: Prognostic value of static cytometry in transitional cell carcinoma of the bladder: recurrence rate and survival in a group of patients at 10 years follow-up. Oncol Rep 15: 213-219, 2006.

16. Taddei I, Piazzini M, Bartoletti R, Dal Canto M and Sardi I: Molecular alterations of E-cadherin gene: possible role in human bladder carcinogenesis. Int J Mol Med 6: 201-208, 2000.

17. Oosterlinck W, Lobel B, Jakse G, Malmström P, Stöckle M and Sternberg C (The EAU Working Group on Oncological Urology): Guidelines on Bladder Cancer. Eur Urol 41: 105-112, 2002.

18. UICC International Union Against Cancer: TNM classification of malignant tumors. 6th edition. Wiley-Liss, New York, pp199-202, 2002 .

19. Mostofi FK, Sobin LH and Torloni H: Histological typing of urinary bladder tumours. World Health Organization, Geneva, 1973. 
20. Bartoletti R, Cai T, Nesi G, Sardi I and Rizzo M: Qualitative and quantitative analysis of angiogenetic factors in transitional cell bladder carcinoma: relationship with clinical course at 10 years follow-up. Oncol Rep 14: 251-255, 2005.

21. Chomczynski P and Sacchi N: Single step method of RNA isolation by acid guanidinium thiocyanate-phenol cloroform extraction. Anal Biochem 162: 156-159, 1987.

22. Sambrook J: Molecular cloning: a laboratory manual. 2nd edition. Cold Spring Harbor Laboratories Press, Cold Spring Harbor, NY, 1989.

23. Pertschuk LP, Feldman JG, Kim DS, et al: Steroid hormone receptor immunohistochemistry and amplification of c-myc protooncogene. Relationship to disease-free survival in breast cancer. Cancer 71: 162-171, 1993.

24. Serdar A, Turhan C, Soner G, et al: The prognostic importance of e-cadherin and p53 gene expression in transitional bladder carcinoma patients. Int Urol Nephrol 37: 485-492, 2005.

25. Takeichi M: Cadherin cell adhesion receptors as a morphogenetic regulator. Science 251: 1451-1455, 1991.

26. Garcia del Muro X, Torregrosa A, Munoz J, et al: Prognostic value of the expression of E-cadherin and beta-catenin in bladder cancer. Eur J Cancer 36: 357-362, 2000.
27. Syrigos KN, Krausz T, Waxman J, et al: E-cadherin expression in bladder cancer using formalin-fixed, paraffin-embedded tissues: correlation with histopathological grade, tumor stage and survival. Int J Cancer 64: 367-370, 1995.

28. Lipponen PK and Eskelinen MJ: Reduced expression of Ecadherin is related to invasive disease and frequent recurrence in bladder cancer. J Cancer Res Clin Oncol 121: 303-308, 1985.

29. Bringuier PP, Umbas R, Schaafsma E, Karthaus HF, Debruyne FM and Schalken JA: Decreased E-cadherin immunoreactivity correlates with poor survival in patients with bladder tumors. Cancer Res 53: 3241-3245, 1993.

30. Mialhe A, Louis J, Montlevier S, et al: Expression of E-cadherin, alfa-, beta- and gamma-catenins in human bladder carcinomas: are they good prognostic factors? Invasion Metastasis 17: 124-137, 1997.

31. Mahnken A, Kausch I, Feller AC and Kruger S: E-cadherin immunoreactivity correlates with recurrence and progression of minimally invasive transitional cell carcinomas of the urinary bladder. Oncol Rep 14: 1065-1070, 2005. 